\title{
Location of valinomycin in lipid vesicles
}

\author{
M. B. SANKARAM and K. R. K. EASWARAN \\ Molecular Biophysics Unit, Indian Institute of Science, Bangalore 560012, India \\ MS received 26 March 1984
}

\begin{abstract}
The location of the cyclododecadepsipeptide, valinomycin in vesicles formed from two synthetic lipids is studied by differential scanning calorimetry, spin-label partitioning electron paramagnetic resonance and $\left[{ }^{1} \mathrm{H}\right]$-nuclear magnetic resonance. The results show that valinomycin is located near the head group region of dipalmitoyl phosphatidyl choline vesicles and in the hydrophobic core of the dimyristoyl phosphatidyl choline vesicles in the liquid crystalline phase.
\end{abstract}

Keywords. Valinomycin; dipalmitoylphosphatidylcholine; dimyristoylphosphatidylcholine; differential scanning calorimetry; electron spin resonance; nuclear magnetic resonance; ionophore-lipid interaction.

\section{Introduction}

Valinomycin, a cyclic dodecadepsipeptide, is a prototype of carriers (Ovchinnikov et al., 1974) which enhance the cation permeability across membranes by a diffusion of the carrier-cation complex from one side of the membrane to the other. Such a diffusion would take place only when the membrane is in the liquid crystalline phase as is confirmed by temperature-dependent black lipid membrane (BLM) steady-state conductance measurements (Boheim et al. 1980). The carrier complexes with the cation at one interface to form a hydrophobic carrier-cation complex. This would be done with efficiency if the local concentration of the carrier at the interface is enhanced by a preferential location of the uncomplexed carrier at that interface.

Free valinomycin in solution adopts solvent dependent conformations (Mayers and Urry, 1972; Patel and Tonelli, 1973) whereas the conformation of its $K^{+}$complex is independent of the nature of the solvent (Davis and Tosteson, 1975). Since the membrane-water interface is similar to the polar organic solvents and the interior can be modelled by nonpolar organic solvents, it is likely that the conformation of valinomycin at the interfaces is different from that in the interior. We report in this paper our results on the location of valinomycin in dimyristoylphosphatidylcholine (DMPC) and dipalmitoylphosphatidylcholine (DPPC) bilayers, both in the uni- and multi-lamellar vesicular forms, using differential scanning calorimetry (DSC), spin-

\footnotetext{
Abbreviations used: BLM, Black lipid membrane; DMPC, dimyristoylphosphatidylcholine; DPPC, dipalmitoylphosphatidylcholine; DSC, differential scanning calorimetry; EPR, electron paramagnetic resonance; EYL, egg yolk lecithin; LBPT, lipid bilayer phase transition; MLV, multilamellar vesicle; NMR, nuclear magnetic resonance; TEMPO, 2,2,6,6-tetramethylpiperidin-1-oxyl; ULV, unilamellar vesicle.
} 
label partitioning electron paramagnetic resonance (EPR) and $\left[{ }^{1} \mathrm{H}\right]$-nuclear magnetic resonance (NMR) techniques.

\section{Experimental}

Valinomycin, L- $\alpha$-DMPC, L- $\alpha$-DPPC, and $\mathrm{D}_{2} \mathrm{O}$ are from Sigma Chemical Company, St. Louis, Missouri, USA. 2,2,6,6,Tetramethylpiperidin-1-oxyl (TEMPO) was synthesized according to standard procedures (Rozantsev, 1970). DSC thermograms were recorded on a Perkin-Elmer DSC-2C instrument calibrated with Indium and Benzene. Typically about $20 \mu \mathrm{L}(50 \mathrm{mg} / \mathrm{mL})$ of the sample was placed in aluminum pans used for volatile samples. The thermograms were recorded with a heating rate of $10 \mathrm{deg} / \mathrm{min}$ and a range of $10 \mathrm{meal} / \mathrm{sec}$. The EPR spectra were recorded on a JEOL-FE3X spectrometer equipped with a variable temperature accessory. A standard flat quartz cell was used for recording the spectra. The EPR spectra were recorded at increasing temperatures in steps of $2^{\circ} \mathrm{C}$. $\left[{ }^{1} \mathrm{H}\right]-\mathrm{NMR}$ spectra were recorded on a Bruker WH 270 spectrometer. The field-frequency lock was obtained from the deuterium signal of the solvent $\mathrm{D}_{2} \mathrm{O}$.

For the DSC experiments, appropriate amounts of lipid and lipid-valinomycin mixtures were dissolved in chloroform. A thin film was formed by evaporating the chloroform by bubbling dry nitrogen gas through the solution. To the vacuum dried thin film, glass-distilled water was added and the dispersion was shaken thoroughly on a vortex mixture above the lipid bilayer phase transition temperature (LBPT) of the lipid.

Samples for the EPR studies were prepared as for DSC experiments except that, all the samples contained $0.1 \%$ of the EPR probe, TEMPO.

The $\left[{ }^{1} \mathrm{H}\right]-\mathrm{NMR}$ studies were done on sonicated unilamellar vesicles (ULVs) whereas the multilamellar vesicles (MLVs) were used for the DSC and EPR experiments. The ULVs were formed by sonicating the MLVs (Bangham et al., 1974) formed in $\mathrm{D}_{2} \mathrm{O}$ as described for DSC samples. The MLV samples were sonicated on a Branson B-12 sonicator till the solution became clear. The sonicated vesicles are $26 \mathrm{~nm}$ in diameter as checked by the lanthanide shift reagent method (Bergelson, 1978). The lipids were not degraded by sonication as verified by thin layer chromatography with 6:9:2.7:5 $\mathrm{CHCl}_{3}: \mathrm{CH}_{3} \mathrm{OH}: 7 \mathrm{M} \mathrm{NH} \mathrm{NH}_{4} \mathrm{OH}$ as the eluent (Szoka and Papahadjopoulos, 1980).

\section{Results}

The interaction of valinomycin with DPPC MLVs was studied by spin-label partitioning EPR (Shimshick and McConnell, 1973). The EPR spectra of TEMPO in DPPC MLVs containing various concentrations of valinomycin were recorded at different temperatures. The TEMPO parameter, $P$ was plotted against temperature and the inflexion point was taken as the phase transition temperature. $P$ was calculated as the ratio of intensity of the TEMPO signal in the lipid bilayer to the sum of the intensities in the lipid bilayer and water (Shimshick and McConnell, 1973). Such graphs for a few DPPC: valinomycin ratios are shown in figure 1. Free DPPC showed the pretransition at $35^{\circ} \mathrm{C}$ and the main transition at $43^{\circ} \mathrm{C}$. The pretransition broadened initially and gradually disappeared as the valinomycin concentration increased. No 


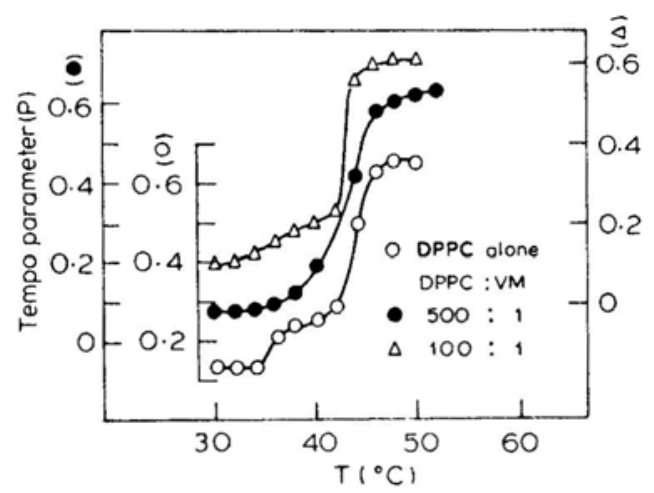

Figure 1. Phase transition temperatures of DPPC-valinomycin MLVs by spin-label partitioning EPR. $[\mathrm{DPPC}]=0.18 \mathrm{M},[\mathrm{TEMPO}]=0.75 \mathrm{mM}$.

significant changes in the melting temperatures of the main transition were noticed.

The lipid bilayer phase transition (LBPT) temperature was followed by DSC also. The thermograms for DPPC alone and with valinomycin at DPPC: valinomycin ratios of 100:1 are shown in figure 2. DPPC showed a main transition at $42^{\circ} \mathrm{C}$ as compared to the literature value of $41.5^{\circ} \mathrm{C}$ (Mabrey and Sturtevant, 1978). As in the case of the EPR experiments, the main transition did not change significantly whereas the pretransition was broadened. The DSC data for the valinomycin-DPPC MLVs have been reported

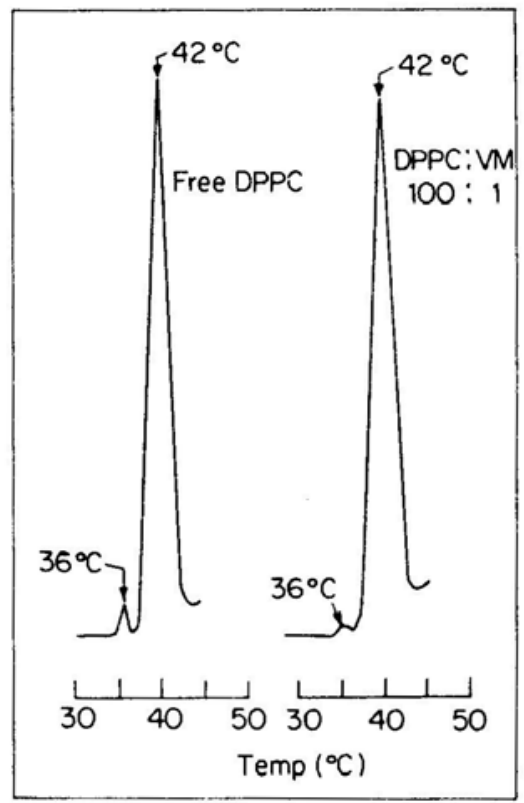

Figure 2. DSC thermograms of DPPC MLVs with valinomycin. [DPPC] $=20 \mathrm{mM}$. 
earlier (Grell et al., 1975) and the results showed that the main transition temperature decreased with increasing addition of valinomycin.

The $\left[{ }^{1} \mathrm{H}\right]-\mathrm{NMR}$ studies were carried out on ULVs from both DMPC and DPPC. To these ULVs, valinomycin was added as a methanolic solution in aliquots of $2 \mu \mathrm{L}$ to obtain the required lipid: valinomycin ratios. Control experiments were performed by adding the same amount of methanol to a portion of the same vesicle solution. No significant changes were observed upon addition of methanol alone.

The line widths of the choline and the aliphatic chain signals of DPPC ULVs were followed by gradual addition of valinomycin at $60^{\circ} \mathrm{C}$, which is above the melting temperature. The $\left[{ }^{1} \mathrm{H}\right]-\mathrm{NMR}$ spectra are shown in figure $3 \mathrm{~A}$. The line widths of the

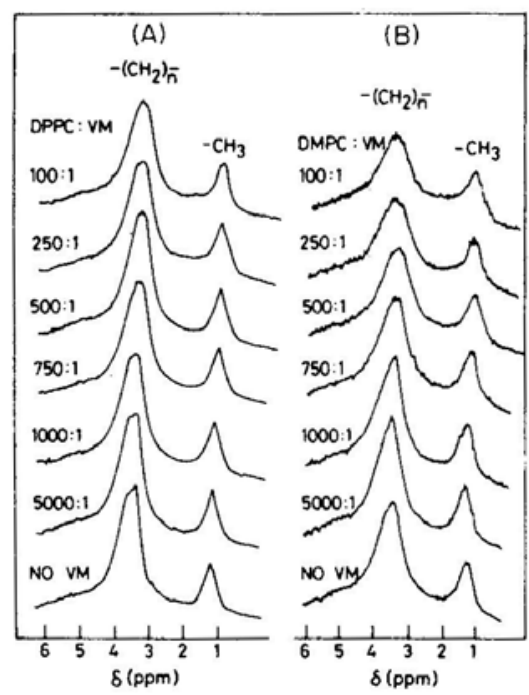

Figure 3. $270 \mathrm{MHz}\left[{ }^{1} \mathrm{H}\right]-\mathrm{NMR}$ spectra (aliphatic chain region) of (A) DPPC ULVs at $60^{\circ} \mathrm{C}$ and (B) DMPC ULVs at $30^{\circ} \mathrm{C}$. [DPPC $]=[\mathrm{DMPC}]=20 \mathrm{mM}$.

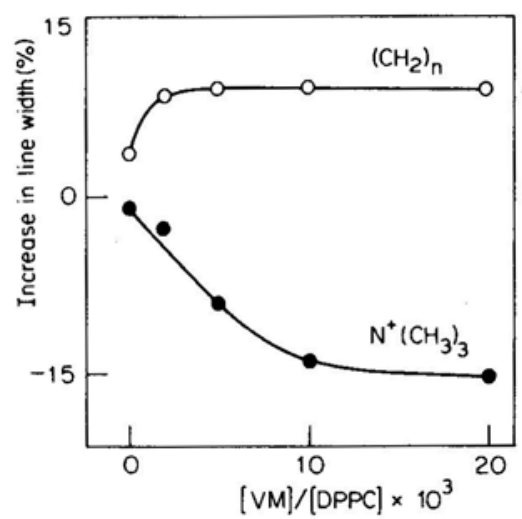

Figure 4. $270 \mathrm{MHz}\left[{ }^{1} \mathrm{H}\right]-\mathrm{NMR}$ titration graph of DPPC-valinomycin ULVs at $60^{\circ} \mathrm{C}$. $[\mathrm{DMPC}]=20 \mathrm{mM}$. 
aliphatic signals showed a slight broadening whereas the head group signals sharpened as valinomycin was gradually added. The titration graphs for the choline and the aliphatic chain signals are shown in figure 4.

The line widths of the choline and the aliphatic chain signals were followed by addition of valinomycin to DMPC ULVs at $30^{\circ} \mathrm{C}$ which is above its melting temperature. The $\left[{ }^{1} \mathrm{H}\right]-\mathrm{NMR}$ spectra are shown in figure $3 \mathrm{~B}$. The titration graphs are shown in figure 5 . In this case, the aliphatic signals showed a significant broadening as valinomycin was gradually added and the choline signal did not show any appreciable changes.

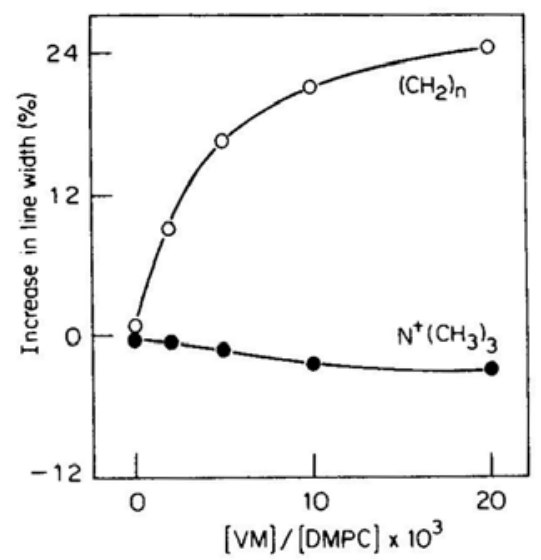

Figure 5. $270 \mathrm{MHz}\left[{ }^{1} \mathrm{H}\right]-\mathrm{NMR}$ titration graph of DMPC-valinomycin ULVs at $30^{\circ} \mathrm{C}$. $[\mathrm{DMPC}]=20 \mathrm{mM}$.

\section{Discussion}

The $\left[{ }^{1} \mathrm{H}\right]-\mathrm{NMR}$ data clearly showed that valinomycin does not effectively penetrate the hydrophobic core of DPPC ULVs. The slight broadening of the methylene proton signal might be due to slower rates of chain rotations (Marsh and Watts, 1981; Chan et al., 1981). The sharpening of the head group choline signals indicated a location of valinomycin near the interface. The head group has been shown to be oriented perpendicular to the bilayer normal with the negatively charged phosphate group interacting with the neighbouring positively charged choline group (Buldt and Wohlgemuth, 1981; Davis, 1983). The sharpening of the choline signal might be due to increased motion of the head group when this interaction is weakened by pulling two lipid molecules apart. A schematic representation of valinomycin in DPPC vesicles is shown in figure 6A. An earlier $\left[{ }^{1} \mathrm{H}\right]$-NMR study of valinomycin-DPPC MLVs has reported similar results (Hsu and Chan, 1973). Valinomycin, in this study, has been proposed to be adsorbed on the membrane surface.

The absence of any changes in the main transition as observed by DSC and EPR confirms the absence of valinomycin in the hydrophobic core of DPPC MLVs as well. The broadening of the pretransition is difficult to explain, because its origin is not well known. The pretransition has been observed in MLVs formed from phosphatidylcho- 

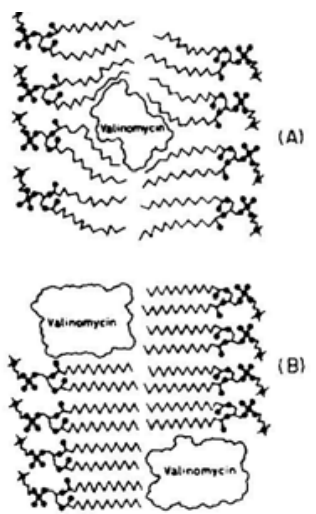

Figure 6. A. Valinomycin in DPPC vesicles. B. Valinomycin in DMPC vesicles.

lines and not from phosphatidylethanolamines (Bergelson, 1978). Based on the DSC data, the pretransition has been associated with conformational rearrangement of the head group portion of the lipid molecule (Chapman, 1975; Janiak et al., 1976,1979), while deuterium quadrupole splitting and $\left[{ }^{31} \mathrm{P}\right]-\mathrm{NMR}$ studies (Gaily et al., 1975) denied such conformational changes at the pretransition. Fluorescence (Jacobson and Papahadjopoulos, 1975) and NMR (McIntosh, 1980; Boroske and Trahms, 1983) studies indicated that the pretransition had a pronounced effect on the arrangement of the acyl chains. The pretransition has not been observed even in large ULVs (Takemoto et al., 1981) which might not form the $P_{\beta}^{\prime}$ structure (Luzzati and Tardieu, 1974). Addition of certain substances like fatty acids (Mabrey and Sturtevant, 1977) and tetraphenylborate to DPPC MLVs caused a disappearance of the pretransition. The presently held opinion is that the pretransition is a consequence of some unknown effects arising from interactions among the bilayer sheets in MLVs.

In the region between the pre and the main transitions ( $P_{\beta}^{\prime}$ phase), the chain tilt has been shown to be less than that in the gel $\left(L_{\beta}^{\prime}\right.$ phase $)$ and it goes to zero in the liquid crystalline phase $\left(L_{\alpha}\right)$ (Pope et al., 1981). Insertion of valinomycin molecules between two lipid molecules might permit a wider range of tilt angles leading to a broadening of the pretransition. A better understanding of the origin of the pretransition is necessary to explain the effects of additives on the pretransition.

Valinomycin interacts with DMPC ULVs in an altogether different way. In this case, the aliphatic chain signals are broadened as valinomycin is added whereas the choline signals do not change significantly. This observation suggests a penetration of valinomycin into the hydrophobic core of the vesicles. The broadening of the methylene signals indicates a lipid aggregation around valinomycin which leads to motional restriction of the lipid chains. Such aggregation has been proposed for valinomycin in egg yolk lecithin vesicles (Walz, 1977, 1979). The location of valinomycin in DMPC vesicles is schematically represented in figure $6 \mathrm{~B}$.

Results from this study and earlier studies are summarized in table 1. These studies indicated that valinomycin is located near the head group in DPPC vesicles and in the 
Table 1. Summary of studies on the location of valinomycin in vesicles.

\begin{tabular}{|c|c|c|c|c|c|}
\hline Lipid & ULV/MLV & $\begin{array}{c}\text { Lipid/ } \\
\text { valinomycin }\end{array}$ & Technique & Location & Reference \\
\hline DMPC & MLV & 24 to 10 & DSC & Interior & Grell et al., (1975) \\
\hline DMPC & ULV & 19 & $C D$ & Interior & Grell et al., (1975) \\
\hline DMPC-d $_{72}$ & ULV & 30 & {$\left[{ }^{1} \mathrm{H}\right]-\mathrm{NMR}$} & Interior & $\begin{array}{l}\text { Feigenson and Meers } \\
\quad(1980)\end{array}$ \\
\hline $\begin{array}{l}\text { DMPC } \\
\text { DPPC }\end{array}$ & $\begin{array}{l}\text { ULV } \\
\text { MLV }\end{array}$ & $\begin{array}{l}1000 \text { to } 10 \\
5000 \text { to } 50\end{array}$ & $\begin{array}{l}{\left[{ }^{1} \mathrm{H}\right]-\mathrm{NMR}} \\
{\left[{ }^{1} \mathrm{H}\right]-\mathrm{NMR}}\end{array}$ & $\begin{array}{l}\text { Interior } \\
\text { Head group region }\end{array}$ & $\begin{array}{l}\text { Present study } \\
\text { Hsu and Chan (1973) }\end{array}$ \\
\hline DPPC & MLV & 1000 to 10 & DSC, EPR & Head group region & Present study \\
\hline DPPC & ULV & 1000 to 10 & {$\left[{ }^{1} \mathrm{H}\right]-\mathrm{NMR}$} & Head group region & Present study \\
\hline EYL & ULV & 125 to 35 & UV & Interior & Walz $(1977,1979)$ \\
\hline EYL & ULV & 10 & $\mathrm{CD}$ & Interior & Grell et al., (1975) \\
\hline
\end{tabular}

hydrophobic core in DMPC vesicles. The location is the same in both MLVs and ULVs. ULVs have been shown to be more disordered than MLVs by Raman spectroscopy (Gaber and Peticolas, 1977), fluorescence (Yguerabide and Foster, 1981) and calorimetry both above and below the melting temperature. The question of the fluidity differences between ULVs from DPPC and DMPC has not been explicitly addressed to in the literature. It appears from the available Raman spectroscopic data (Gaber and Peticolas, 1977) that DMPC vesicles are more disordered than DPPC vesicles, both in MLV and ULV forms. Valinomycin may be able to penetrate the DMPC vesicles which are more disordered than DPPC vesicles.

\section{Acknowledgements}

We thank the University of Hyderabad for use of the JEOL FE3X instrument and Mr. B. P. Shastri for the synthesis of TEMPO. M.B.S. thanks the University Grants Commission, New Delhi for a fellowship.

\section{References}

Bangham, A. D., Hill, M. W. and Miller, N. G. A. (1974) Meth. Membrane Biol., 1, 1.

Bergelson, L. D. (1978) Meth. Membrane Biol., 9, 275.

Boheim, G., Hanke, W. and Eibl, H. (1980) Proc. Natl. Acad. Sci. USA, 77, 3403.

Boroske, E. and Trahms, L. (1983) Biophys. J., 42, 275.

Buldt, G. and Wohlgemuth, R. (1981) J. Membrane Biol., 58, 81

Chan, S. I., Bocian, D. F. and Petersen, W. O. (1981) in Membrane Spectroscopy, ed. E. Grell, (Berlin: Springer-Verlag).

Chapman, D. (1975) Biochemistry, 7, 1.

Davis, D. G. and Tosteson, D. C. (1975) Biochemistry, 14, 3962.

Davis, D. H. (1983) Biochim. Biophys. Acta, 737, 117.

Feigenson, G. W. and Meers, P. R. (1980) Nature (London), 283, 313.

Gaber, B. P. and Peticolas, L. (1977) Biochim. Biophys. Acta, 465, 260. 
Gally, H. U., Niederberger, W. and Seelig, J. (1975) Biochemistry, 14, 3647.

Grell, E., Funck, Th. and Eggers, F. (1974) in Membranes, A Series of Advances, (ed. G. Eisenman) (NewYork: Dekker) vol. 3, Chapter 1.

Hsu, M. C. and Chan, S. I. (1973) Biochemistry, 12, 3872

Jacobson, K. and Papahadjopoulos, D. (1975) Biochemistry, 14, 152..

Janiak, M. J., Small, D. M. and Shipley, G. G. (1976) Biochemistry, 15, 4576.

Janiak, M. J., Small, D. M. and Shipley, G. G. (1979) J. Biol. Chem., 254, 6068.

Luzzati, V. and Tardieu, A. (1974) Ann. Rev. Phys. Chem., 25, 79.

Mabrey, S. and Sturtevant, J. M. (1977) Biochim. Biophys. Acta, 486, 444.

Mabrey, S. and Sturtevant, J. M. (1978) Meth. Membrane Biol., 9, 237.

Marsh, D. and Watts, A. (1981) in Liposomes: From Physical Structure to Therapeutic Applications', ed. C. G. Knight, (Amsterdam: Elsevier).

Mayers, D. F. and Urry, D. W. (1972) J. Am. Chem. Soc., 94, 77.

Mclntosh, T. J. (1980) Biophys. J., 29, 237.

Ovchinnikov, Yu.A., Ivanov, V. T. and Shkrob, A. M. (1974) Membrane Active Complexones, (Amsterdam: Elsevier).

Patel, D. J. and Tonelli, A. E. (1973) Biochemistry, 12, 486.

Pope, J. H., Walker, L., Cornell, B. A. and Francis, G. W. (1931) Biophys. J., 35, 509.

Rozantsev, E. G. (1970) Free Nitroxyl Radicals, (New York: Plenum Press).

Shimshick, M. and McConnell, H. M. (1973) Biochemistry, 12, 2351.

Szoka, F. and Papahadjopoulos, D. (1980) Ann. Rev. Biophys. Bioeng., 9, 467.

Takemoto, H., Inoue, S., Yasunaga, T., Sukigara, M. and Toyoshima, Y. (1981) J. Phys. Chem., 85, 1032. Walz, D. (1977) in Bioenergetics of Membranes, ed. L. Packer, (Amsterdam: Elsevier).

Walz, D. (1979) Chimia, 33, 45.

Yguerabide, J. and Foster, M. C. (1981) in Membrane Spectroscopy, (ed. E. Grell) (Berlin: Springer-Verlag). 